\title{
La perspectiva de género y su incidencia en el aprovechamiento matemático
}

\author{
Sáenz Sánchez Berna Karina \\ Esc. 24 de Febrero No. 2645, SECyD \\ bernasaenz@hotmail.com
}

\begin{abstract}
Resumen
La perspectiva de género va cobrando auge en nuestro tiempo, como una alternativa de igualdad entre hombres y mujeres; a pesar de ello, existe tendencia a considerar que los hombres y las mujeres presentan diferencias significativas en el desarrollo lingüístico y las habilidades matemáticas. El propósito de esta investigación fue determinar si hay diferencia entre hombres y mujeres en el desarrollo de estas habilidades, partiendo de la premisa de que las mujeres desarrollan mejor las habilidades lingüísticas y los hombres habilidades matemáticas. Para ello se realizaron dos estudios, en el primero se parte de las creencias que manejan los docentes con respecto al género y en el segundo se aplicó el Test WISC-R a 486 alumnos de la zona 92 del nivel primaria, para establecer la diferencia en habilidades lingüísticas y matemáticas entre hombres y mujeres, obteniendo como resultado que las mujeres desarrollan más el área lingüística, los hombres la ubicación espacial, pero no se mostró diferencia en el desarrollo de habilidades matemáticas.
\end{abstract}

Palabras clave

Habilidades lingüísticas, habilidades matemáticas, ubicación espacial.

\section{Introducción}

El aprendizaje es un elemento inherente de la vida en todos sus ámbitos. Se aprende de la experiencia y de los procesos de enseñanza intencionales o espontáneos que se sitúan en la familia, escuela y en las demás instituciones de la organización social que forma parte del contexto del individuo.

La escuela como institución es el principal agente encargado de educar, teniendo como sustento un diseño curricular. La propuesta curricular que propone los planes y programas de estudio presenta una serie de contenidos programáticos a desarrollar a lo largo de la escuela, mediante una dosificación por asignaturas. Entre dichas asignaturas destacan por su importancia, la de español y la de matemáticas; consideradas como punto de partida en evaluaciones nacionales e internacionales y determinar la calidad de la educación en el nivel básico.

De acuerdo a los resultados presentados por la prueba ENLACE (Evaluación Nacional de Logros Educativos en Centros Escolares) aplicada en el 2008 se observa que $61.4 \%$ de los alumnos se ubican en el nivel elemental, $21 \%$ en el rango suficiente, el $16 \%$ en el nivel bueno y sólo el $1.6 \%$ en el nivel de 
excelente, lo anterior manifiesta un quebrante en el aprendizaje de habilidades matemáticas en alumnos de nivel elemental. Con respecto a las habilidades lectoras el $20.7 \%$ en el nivel suficiente, el $58.1 \%$ de los participantes se ubican en el nivel elemental, el $19.6 \%$ se encuentra en el nivel bueno y sólo el 1.7\% destaca en el nivel de excelente (SEP, 2010).

En evaluaciones internacionales como PISA (Programa Internacional de Evaluación de Estudiantes) que evalúa ciencia, lectura y matemáticas en el 2006, México se ubicó en el lugar 49 en ciencias, 48 en matemáticas y 43 en lectura. Esto orilla al planteamiento ¿la escuela está satisfaciendo las necesidades educativas de aprendizaje de los alumnos?

Esta problemática en la baja eficiencia de resultados nacionales e internacionales conlleva a pensar en una serie de factores estructurales y contextuales que limitan el trabajo dentro de las aulas, aunado a las condiciones socioeconómicas, cognitivas y de género que afectan la calidad educativa.

En el presente documento se muestran los resultados obtenidos al trabajar con una investigación de corte mixto, es decir cualitativo y cuantitativo, mediante la recolección de creencias de los docentes en una encuesta y a través de la aplicación de un instrumento estandarizado (WISC-R) para los estudiantes de nivel primaria. Los resultados, muestran que las creencias de los docentes con respecto al desarrollo de habilidades matemáticas en sus estudiantes son en función de su sexo y los resultados obtenidos del test, muestran que tal como lo sustenta la teoría las mujeres desarrollan las habilidades lingüísticas de una mejor manera con respecto a los hombres, que los hombres desarrollan mejora la ubicación espacial, pro no se encontraron elementos que pongan de manifiesto una diferencia significativa entre hombres y mujeres con respecto al desarrollo de habilidades matemáticas.

\section{Conceptualización teórica}

Schmelkes (1996), sostiene que la calidad educativa es un concepto muy complejo, siendo necesario para su definición tomar en cuenta diversos aspectos que ayuda a mejorar la educación, como la oferta real que se ofrece a la sociedad, eficiencia en el logro de los objetivos previstos por la currícula, la equidad educativa y la eficiencia en el logro de los resultados; si no se cumple con estos elementos surge el fracaso escolar.

Un elemento condicionante del fracaso escolar es el bajo rendimiento del alumno en una materia en específico. Para García (2001), el bajo rendimiento es un curso es una consecuencia de la falta de interés que presenta el alumno, la poca motivación por parte del maestro y los problemas emocionales o baja autoestima que puede presentar el educando.

En términos evaluativos, el Instituto Nacional para la Evaluación de la Educación (INEE, 2004) considera que cuando los alumnos permanecen en el sistema con un rendimiento inferior al promedio, están presentando bajo rendimiento lo cual contribuye a disminuir, la calidad de la educación.

$$
\text { Con respecto al bajo }
$$
aprovechamiento en la asignatura de matemáticas, Saint-Onge (2000), expone que se debe a los procedimientos de enseñanza que se han orientado principalmente a la memorización más que a la comprensión de las reglas del pensamiento lógico-matemático, al poco gusto que muestra el docente por impartir 
la asignatura, a la inadaptación curricular, al desinterés de los alumnos hacia la materia y las diferencias de aprendizaje que se llevan a cabo entre los mismos alumnos.

Estas diferencias de aprendizaje se han visto a nivel mundial, mediante una comparación del aprovechamiento que logran los niños y las niñas en las pruebas aplicadas. De acuerdo al Fondo de las Naciones para la Infancia (INICEF, 2004), hay diferencias en el aprendizaje entre hombres y mujeres, situación que es más evidente en los contextos socioeconómicos desfavorecidos. En América Latina y el Caribe, los varones presentan mayores tasas de repetición y rendimiento académico en comparación con las niñas.

Hay que reconocer que aunque en nuestro tiempo se pregona una educación de igualdad, rara vez se ve garantizada en la práctica concreta educativa. En este sentido, Fainhloc (1994) expone:

Si bien no se hace distinción alguna a los objetivos pedagógicos entre uno y otro sexo, los textos y materiales didácticos, contenidos, estrategias de enseñanza y evaluación, implican valores que ponen en primer plano al papel familiar de la mujer, sin requerir de ellas otras responsabilidades potenciales o en el mejor de los casos reproducen para la mujer las expectativas sociales ocupacionales $\mathrm{y}$ domésticas (p.51).

Ante esto, se hace necesario plantear una cuestión esencial: ien dónde se inicia esta diferenciación y exclusión sexual dentro del proceso de enseñanza y aprendizaje de las matemáticas? La respuesta es compleja, por lo que es necesario analizar diversas perspectivas que van desde la biológica hasta la sociocultural sin dejar de lado la visión psico-educativa.

Desde el punto biológico, una de las primeras diferencias que se hacen entre hombres y mujeres parte de su condición sexual. Para López (2003), el sexo se ubica en el plano eminentemente biológico que no es susceptible a cambio y que se constituye de las atribuciones fisiológicas y anatómicas del individuo.

En las aportaciones de Blum (1997) se encuentra que existen diferencias en el funcionamiento y la estructura cerebral entre hombres y mujeres, debido al tamaño del cerebro, el cual es mayor en los varones pero en las mujeres el ritmo de envejecimiento cerebral es más lento.

Estudios llevados a cabo por Rogers (2001) exponen que los niveles desiguales de testosterona en los sujetos se asocian de manera diferente en las tareas espaciales, donde se explica que el razonamiento matemático está relacionado con los niveles de testosterona en hombres, aunque no en mujeres. Sin embargo, los niveles de estrógeno en las mujeres se asocian con cambios en la fluidez verbal, velocidad perceptiva y destreza manual.

Liaño (1998) hace una diferenciación neurológica del cerebro entre hombres y mujeres, determinando que el cerebro masculino está mejor dotado para el cálculo, aritmética y cuestiones espaciales; en tanto el cerebro femenino está mejor dotado para el procesamiento del lenguaje (fluidez verbal) y la inteligencia emocional, al ser más capaz de intuir el estado afectivo y emocional de los interlocutores.

Pease (2002) considera que en la estructura cerebral, el sexo juega un papel importante en la formación de la conducta. Los cerebros masculino y femenino no son idénticos; el hombre tiene más desarrollada ciertas áreas como la del sentido espacial, mientras que el cerebro femenino posee más conexión entre los hemisferios, lo que explica su mayor facilidad para el lenguaje. 
Estas explicaciones en la diferencia estructural del cerebro a partir del plano biológico se ponen a la vez de manifiesto en las diferencias que se encuentran entre hombres y mujeres desde la perspectiva psicoeducativa.

Whyte (1987) manifiesta que la escuela es un lugar propicio para desarrollar en las niñas un rol de conducta femenina $y$ a los hombres un rol masculino. Además, la perspectiva de padres y maestros son diferentes, ya que se espera que los hombres presenten mejores resultados en aprendizaje cognitivo y las mujeres en conducta $\mathrm{y}$ obediencia.

Miguez (2003) sostiene que hasta ahora, la escuela transmite un sistema de género con carácter androcéntrico, es decir, propone una jerarquía de géneros que sitúa a la mujer en un plano marcadamente inferior al hombre. Esta desigualdad se podría reflejar en la manera como el docente se conduce al actuar como mediador entre los planteamientos propuestos en el currículum nacional y las necesidades y perspectivas del alumno.

$$
\text { Para ello, González (2000) }
$$
considera que el profesor es el que reafirma las diferencias de enseñanza por sexo, al considerar que los intereses son diferentes; lo que conlleva a una interacción y trato diferenciado, donde las expectativas que se tienen por sexo son muy importantes.

Según Whyte (1987) el profesorado considera que los niños tienen más dificultades en la lectura en los primeros años escolares y que las niñas la tienen en matemáticas y ciencia. En tanto Dumais (2002), expresa que los niños y las niñas llegan al colegio a los 5 o 5 años con destrezas similares, pero las niñas tienen un mejor lenguaje y los niños tienen un mejor rendimiento de relación espacial. Diversos investigadores (SuetoLing Pong 1997, Lummis y Stevenson 1990, Fainhloc 1994, Feingold 1988, González 2004) han encontrado diferencias en el aprendizaje de las matemáticas y las cuestiones verbales entre hombres y mujeres, sin embargo, se manifiesta que la brecha que separa estas diferencias a partir del género cada días se están acortando, debido a que las diferencias de aprendizaje en estos rubros se dan en función de las condiciones de enseñanza.

Aunado a ello, se encuentran las condiciones socio-culturales que están presentes en estas diferenciaciones. Por un lado se tiene el papel que juega la mujer en la sociedad el cual es atribuido por el trabajo manual que debe de llevar a cabo y por el otro lado está el papel intelectual que realiza el hombre. De acuerdo con Ander (1972), es necesario que la mujer deje de caracterizarse solamente por su imagen materna y emotiva, ya que esta concepción la lleva a ser menos intelectual y lógica que el hombre y a la vez más intuitiva que él.

Esta concepción, lleva a pensar que los estereotipos de género se manifiestan como realidades subjetivas, teniendo como consecuencia que las matemáticas sean patrimonio del hombre (Fainhloc, 1994).

Lo anterior permite plantear diversas preguntas para ser investigadas: ¿cuáles son las ideas que expresan los docentes de educación básica sobre las semejanzas y diferencias que tienen los niños y niñas en su rendimiento escolar en matemáticas? ¿cuáles son las semejanzas y diferencias que se manifiestan entre los profesores y profesoras, en relación con la manera como enseñan competencias en los niños y niñas? Y ¿existen diferencias 
entre alumnos y alumnas en habilidades matemáticas y lingüísticas como una función de su género?

\section{Metodología \\ Estudio 1}

Para conocer las ideas que expresan los docentes de educación básica sobre las diferencias que tienen los niños y niñas en el rendimiento escolar y qué diferencias se presentan en los maestros y las maestras de este nivel en la enseñanza de las matemáticas se llevó a cabo un estudio de tipo exploratorio, en el cual participaron 40 docentes de educación primaria de 7 escuelas de las ciudades de Chihuahua y Santa Eulalia elegidos por medio de un muestreo experimental y aplicando la técnica de la encuesta y la entrevista. El $77.5 \%$ fue del sexo femenino y el resto del sexo masculino, $20 \%$ con estudios de Normal Básica, $67.5 \%$ con nivel licenciatura, $10 \%$ con maestría y $2.5 \%$ omitió este dato. Los años de servicio tuvieron un rango mínimo de un año y un máximo de 29 años don una ds=1.66 y una media de 12 años de servicio.

A todos los participantes se les hicieron tres preguntas abiertas, a 23 de ellos (57.5\%) se les aplicó la encuesta y al resto 17 (42.5\%) mediante una entrevista. Las preguntas se hicieron con la finalidad de conocer las ideas que expresan los docenes sobre: a) rendimiento, b) el aprendizaje y c) la enseñanza de las matemáticas considerando las diferencias entre niños y niñas. Las respuestas se organizaron en categorías conforme a su frecuencia, utilizando para ello, la técnica sugerida por Ajzen y Fishber (1997, 1982).

Las respuestas se analizaron en dos etapas. En la primera se escribieron todas las respuestas que los participantes expresaron y posteriormente se agruparon por similitud semántica, de tal forma que cada expresión tuviera un sentido único. En la segunda etapa, se listaron todas las expresiones y se registraron las frecuencias en que se mencionó cada una. Luego se colocaron en orden descendente $\mathrm{y}$, en una tabla, se indicaron sus valores de frecuencia, frecuencia relativa $y$ frecuencia acumulada.

Considerando el valor de frecuencia relativa acumulada, se eligieron las expresiones que agruparan del 75 al $80 \%$ de todas las respuestas, conforme al criterio sugerido por Ajzen y Fishbern (1972, 1982), los resultados obtenidos fueron los siguientes: 
RECIE. Revista Electrónica Científica de Investigación Educativa Vol. 1, núm. 2, enero-diciembre 2013, pp. 373-383.

Tabla 1. Respuestas a la pregunta: ¿Qué diferencias encuentra en el rendimiento de matemáticas entre hombres y mujeres?

\begin{tabular}{|c|c|c|c|}
\hline AFIRMACION & $\mathrm{F}$ & FR & $\begin{array}{c}\mathrm{FR} \\
\mathrm{A}\end{array}$ \\
\hline Es igual entre ambos, ya que tienen la misma capacidad de razonamiento & 6 & $\begin{array}{c}15 . \\
8\end{array}$ & 15.8 \\
\hline Por dedicación, interés y comprensión las mujeres aprenden más que los hombres & 6 & 8 & 31.6 \\
\hline Las mujeres tienen mayor rendimiento en matemáticas que los hombres & 5 & 13. & 44.7 \\
\hline Las mujeres tienen más altos índices de reprobación & 4 & $\begin{array}{c}10 . \\
5\end{array}$ & 55.3 \\
\hline El hombre tiene más facilidad para aprender matemáticas & 3 & 7.9 & 63.2 \\
\hline Hombres y mujeres tienen las mismas posibilidades y limitaciones de aprendizaje & 3 & 7.9 & 71.1 \\
\hline No hay diferencia de género, el plan de estudio no tiene un enfoque sexista & 3 & 7.9 & 78.9 \\
\hline Otras expresiones & 7 & $\begin{array}{c}18 . \\
4\end{array}$ & 21.1 \\
\hline Total & $\begin{array}{l}3 \\
8\end{array}$ & 100 & 100 \\
\hline
\end{tabular}

Los simbolos de las columnas significan: F= Frecuencia, FR= Frecuencia Relativa y FRA= Frecuencia Relativa Absoluta

En tanto la segunda pregunta ¿Qué diferencias encuentra en la manera de aprender matemáticas entre hombres $\mathrm{y}$ mujeres? Conformó el grupo de aprendizaje matemático, el cual también fue analizado de manera cualitativa mediante el agrupamiento de expresiones dadas por los participantes. Los resultados obtenidos fueron los siguientes: 
Tabla 2. Respuestas a la pregunta ¿Qué diferencias encuentra en la manera de aprender matemáticas entre hombres y mujeres?

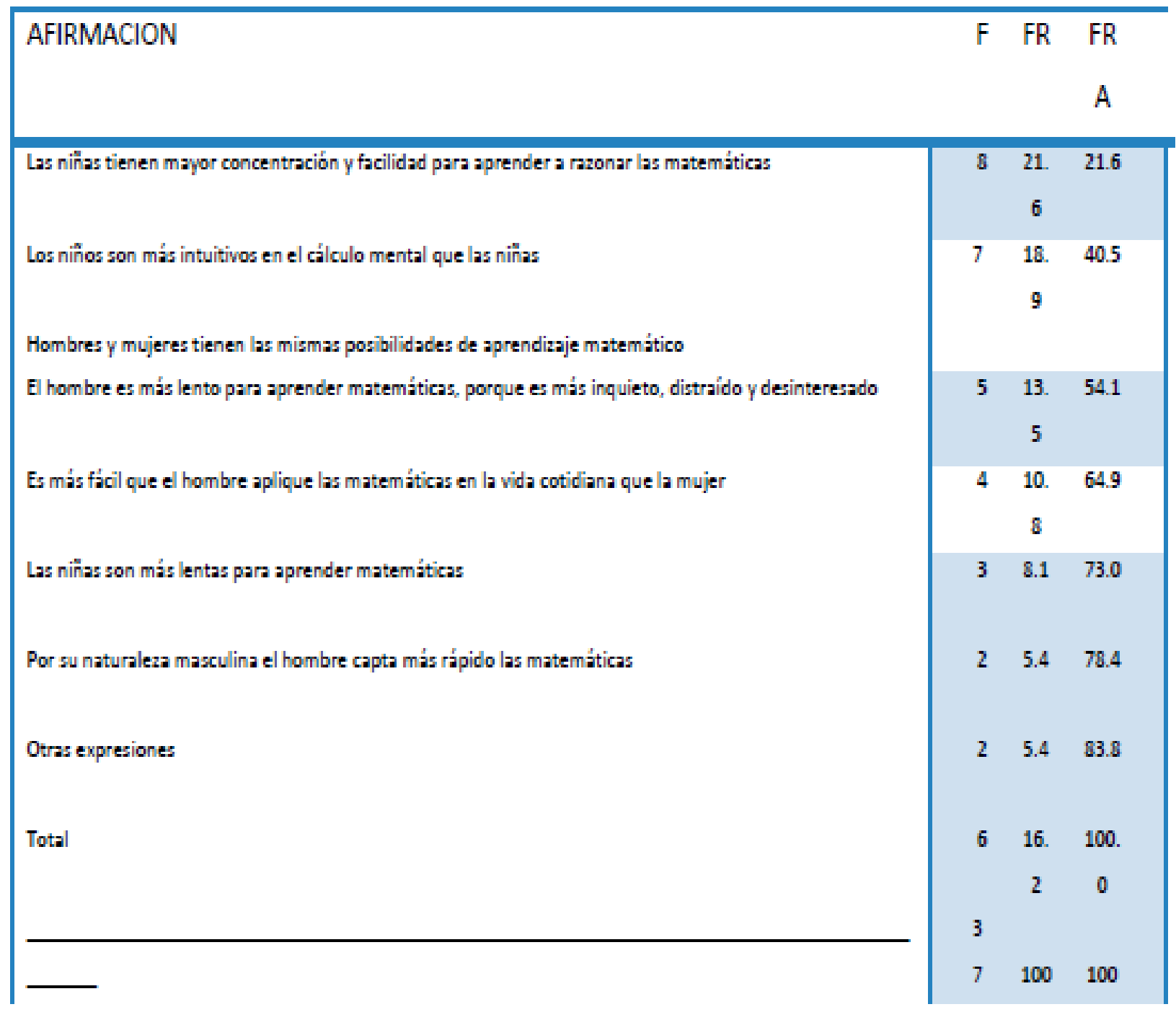

Por su parte, la pregunta 3 ¿Qué diferencia(s) encuentra en la manera de cómo enseña la/el maestra(o)

matemáticas a hombres y mujeres?, se obtuvieron los siguientes resultados: 
Tabla 3. Respuesta a la pregunta: ¿Qué diferencias encuentra en la manera de cómo enseña el/la maestra(o) matemáticas a hombres y mujeres?

\begin{tabular}{|c|c|c|c|}
\hline AFIRMACION & $\mathrm{F}$ & $\mathrm{FR}$ & $\begin{array}{c}\mathrm{FR} \\
\mathrm{A}\end{array}$ \\
\hline El docente planea las mismas actividades para hombres y mujeres & 8 & $\begin{array}{c}21 . \\
2\end{array}$ & 21.2 \\
\hline Las maestras son más pacientes y creativas para enseñar las matemáticas & 6 & $\begin{array}{c}16 . \\
7\end{array}$ & 38.9 \\
\hline Se planea en forma general, no hay un estilo para enseñar primero a las nin̈as y luego a los niños & 6 & $\begin{array}{c}16 . \\
7\end{array}$ & 55.6 \\
\hline La maestra es más meticulosa, el maestro más práctico en la enseñanza de las matemáticas & 3 & 8.3 & 63.9 \\
\hline El aprendizaje depende del esfuerzo del docente por impartir la asignatura de matemáticas y no del género & 2 & 5.6 & 69.4 \\
\hline \multirow[t]{2}{*}{$\begin{array}{l}\text { La diferencia de aprendizaje está en la manera de cómo el docente aborda la materia, no del género del } \\
\text { estudiante }\end{array}$} & 2 & 5.6 & 75.0 \\
\hline & 2 & 5.6 & 80.6 \\
\hline \multicolumn{4}{|l|}{ El profesor se interesa más por el resultado del aprendizaje, la maestra se centra en el procedimiento } \\
\hline & 2 & 5.6 & 86.1 \\
\hline \multicolumn{4}{|l|}{ Más que el género influyen la edad, material didáctico, estrategias y trabajo del docente en enseñanza de } \\
\hline matem. & 5 & $\begin{array}{l}13 . \\
9\end{array}$ & 100 \\
\hline \multirow[t]{2}{*}{ Otras expresiones } & 3 & & 100 \\
\hline & 6 & 100 & \\
\hline Total & & & \\
\hline
\end{tabular}

\section{Discusión del estudio 1}

Como se puede observar en la primera pregunta, la categoría con mayor número de respuestas denota que no hay diferencia entre niños y niñas para el aprovechamiento de las matemáticas, pero si se consideran las dos respuestas descendientes se considera que las mujeres tienen un mayor aprovechamiento en matemáticas que los hombres.

En la segunda tabla se observa que 
la categoría con mayor frecuencia manifiesta que las niñas tienen más alto nivel de aprendizaje de matemáticas que los niños, en tanto las dos siguientes preguntas en frecuencia arrojan que el niño tiene más alto nivel de aprendizaje o que ambos (niños y niñas) tienen las mismas posibilidades de aprendizaje.

En la tercer tabla se detecta que la categoría con mayor frecuencia indica que no hay diferencia en la enseñanza, ya que se planea de manera general, pero en las respuestas siguientes hay una clara evidencia que compara el trabajo de maestras y maestros, donde la maestra se considera más creativa y paciente para enseñar matemáticas, las maestras y los maestros atienden de manera individual a quien tenga problema con la materia.

Lo anterior permite determinar que hay elementos que ponen de manifiesto que el docente si tiene una perspectiva de género con respecto a la enseñanza de las matemáticas, ya que la mayoría de las maestras, coinciden en que la mujer aprende más fácilmente la asignatura y los maestros consideran que el niño le lleva la delantera a la niña.

\section{Estudio 2}

El presente estudio tiene como propósito el comprobar si el género es una condición determinante que muestra diferencias en el desarrollo de habilidades lingüísticas y matemáticas entre hombres y mujeres de educación básica. Basándose en las ideas manejadas por Pérez (2002):

El estudio psicológico de las diferencias sexuales en habilidades cognitivas informa de una superioridad masculina en habilidad matemática $\mathrm{y}$ espacial y una superioridad femenina en habilidades verbales

Desprendiendo la siguiente hipótesis de investigación:

"El sexo es un factor determinante en el desarrollo de habilidades matemáticas, habilidades lingüísticas y ubicación espacial de los y las estudiantes de nivel primaria", pretendiendo determinar si el aprovechamiento matemático de los niños es mayor que el de las niñas. Planteando la siguiente pregunta: ¿Existen diferencias entre alumnos $\mathrm{y}$ alumnas en habilidades matemáticas y lingüísticas como una función de su género?

Para ello, participaron 486 sujetos pertenecientes a 8 escuelas del nivel básico de la Zona 92 de la cd. de Chihuahua; los estudiantes cursaban de $4^{\circ}$ a $6^{\circ}$ grado y con una edad fluctuante entre los 8 y 15 años. De los 486 participantes, 243 (50\%) fueron del sexo masculino y 243 (50\%) del sexo femenino, 2 participantes eran de 8 años, 31.1\% de 9 años, el grueso de los participantes fue de 10 años (32.5\%), 42 participantes fluctuaron entre los 12 y 14 años y solo 1 de 15 años.

Para corroborar la hipótesis se les aplicaron tres subtest, de la escala de Weschler para niños, revisada (WISC-R, 1974), el cual contó con 44 reactivos de las cuales 4 son variables sociodemográficas, 14 para medir habilidades matemáticas a través del planteamiento oral de problemas aritméticos, 17 reactivos para medir las habilidades lingüísticas, específicamente de comprensión de preguntas planteadas de manera oral y el resto de los reactivos fueron para medir ubicación espacial mediante la resolución de 8 laberintos.

Para determinar si se presentaron diferencias con respecto al desarrollo de habilidades matemáticas, lingüísticas $\mathrm{y}$ ubicación espacial entre niños y niñas de educación primaria, se hizo un análisis descriptivo con el Statistic Pachege for Social Sciences (SPSS) de las respuestas obtenidas por el total de variables, 
agrupadas en: total de habilidades matemáticas, total de habilidades lingüísticas y total de ubicación espacial; para obtener las medidas de tendencia central y de variabilidad.

Posteriormente se hizo un análisis mediante la homogeneidad de la varianza para determinar la significatividad de cada total de variables agrupadas, con un grado de libertad para cada uno de los grupos. Por último se realizó un análisis factorial de varianza para determinar mediante la suma de los resultados obtenidos por grupo (masculino y femenino) el grado de significatividad que había en las diferencias entre uno y otro grupo, cuyo criterio es que fuera $<.05$.

Tabla 4. Comparación de medias en el total de habilidades evaluadas. ( $M=$ media, $D E=$ desviación estándar, F= Razón de Fisher, La prueba de Levene es la información sobre la homogeneidad de la varianza y la probabilidad de independencia al azar)

\begin{tabular}{|l|l|l|l|l|l|l|l|}
\hline & \multicolumn{2}{|l|}{ Hombres (N=243) } & \multicolumn{2}{l|}{ Mujeres (N=243) } & \multicolumn{2}{l|}{ Comparación } \\
\hline Afirmaciones & $\mathrm{M}$ & $\mathrm{DE}$ & $\mathrm{M}$ & $\mathrm{DE}$ & $\begin{array}{l}\text { Prueba } \\
\text { de } \\
\text { Levene }\end{array}$ & $\mathrm{F}$ & $\begin{array}{l}\text { Probabilidad } \\
\text { de error tipo } \\
\mathrm{I}\end{array}$ \\
\hline $\begin{array}{l}\text { Total de habilidades } \\
\text { matemáticas }\end{array}$ & 8.6 & 2.2 & 8.3 & 1.9 & .161 & 1.97 & $\mathrm{p} .05$ \\
\hline $\begin{array}{l}\text { Total de habilidades } \\
\text { linguísticas }\end{array}$ & 13.1 & 3.6 & 13.8 & 3.6 & .023 & 5.18 & $<.05$ \\
\hline $\begin{array}{l}\text { Total de ubicación } \\
\text { espacial }\end{array}$ & 20.8 & 3.3 & 19.9 & 1.1 & .021 & 5.32 & $\mathrm{p}<.05$ \\
\hline
\end{tabular}

La tabla anterior permite visualizar que no se observar una diferencia significativa entre el desarrollo de habilidades matemáticas entre hombres y mujeres, ya que la significatividad fue de $\mathrm{p}>.161$.

En cuanto a las habilidades lingüísticas se obtuvo un índice de significatividad arrojado por el análisis de varianza comparativo con un grado de libertad fue de $\mathrm{p}<.023$ y una razón $(F)$ de 5.18 , lo cual muestra una diferencia en el desarrollo de habilidades lingüísticas entre sexos, favoreciendo con ello a las mujeres, ya que son ellas quienes tienen más alto desarrollo en este rubro.

Con respecto a la ubicación espacial, se obtuvo que los hombres presentaron un puntaje más alto en este subtest, de acuerdo al análisis de varianza, con un grado de libertad, se obtuvo una razón (F) de 5.322 y una significatividad de $\mathrm{p}<.021$, es decir, los hombres presentan mayor ubicación espacial con respecto a las mujeres.

\section{Discusión}

El estudio anterior fue crucial en la investigación para contrastar los 
supuestos teóricos sustentados en el marco teórico y determinar las diferencias en habilidades lingüísticas y matemáticas que presentan hombres y mujeres; donde de acuerdo a los datos obtenidos no hay una diferencia en el razonamiento matemático entre hombres y mujeres, más no así en el desarrollo de habilidades lingüísticas en las cuales se ven favorecidas las mujeres, tal como lo manifiesta Liaño (1998) quien sostiene que las mujeres tienden a desarrollar las habilidades lingüísticas (procesamiento del lenguaje y fluidez verbal).

En tanto en ubicación espacial se tuvo una significatividad que favoreció a los hombres, confirmando con ello lo dicho por Feingold (1988) los hombres tienen una superioridad en mecánica y visión espacial y Liaño (19998) quien manifiesta que por su estructura cerebral el hombre tiende a desarrollar más las áreas de aritmética, cálculo y visión espacial

\section{Referencias}

Ander, E. y otros (1972) Opresión y marginalidad de la mujer. Buenos Aires: Humanitas.

Fainholc, B. (1994) Hacia una escuela no sexista. Buenos Aires: Aique.

Feingold, A. (1988) Cognitive Gender Differences Are Disappearing. American Psychologist, vol. 43, No. 2, p. 95-103. February 1998.

García, P.M. (2011) Fracaso escolar en educación primaria y secundaria y trastorno por déficit de atención con hiperactividad. Madrid: Bilbao

González, J. (2004) Género y matemáticas: balanceando la ecuación. México:
Porrúa/UPN.

González, J. (2000) Políticas públicas en material de género y educación: análisis del caso mexicano. En: Construyendo la diversidad de nuevas orientaciones en género $\mathrm{y}$ educación. México: Porrúa.

INEE (2004) Resultados de la prueba Pisa 2003 y 2004 en México

Liaño, H. (1998) Cerebro de hombre, cerebro de mujer. Barcelona: Ediciones.

López, L. (2003) ¿Te asusta el feminismo? En: 10 años de la ACU. p. 7-12 México: Asociación Colimense de Universitarios.

Miguez, F. (2003) Género y estilos de aprendizaje en secundaria. Documento: resumen de reporte de investigación. Estudios de género en educación. México: UPN.

Pease, A. y Pease, B. (2003) Por qué los hombres y las mujeres somos tan diferentes. Dos sexos en lucha. Muy interesante, año XX, No. 5, p.74-78. Mayo 2003.

Roger, L. (2001) Sexing the brain. Columbia University, New York

Saint-Onge, M. (2000) Yo explico pero ellos...¿aprenden? México: SEP/FCE.

Schmelkes, S. (1996) La evaluación de los centros escolares. Presentación en el taller sobre Evaluación docente y de centros educativos. México: CINESTAV/IPN/SEP.

Whyte, J. (1987) La educación de lo femenino. Barcelona: Aliorna. 
RECIE. Revista Electrónica Científica de Investigación Educativa Vol. 1, núm. 2, enero-diciembre 2013, pp. 373-383. 